\title{
Per capita sugar consumption is associated with severe childhood asthma: an ecological study of 53 countries
}

\author{
*Simon Thornleya, Alistair Stewarta, Roger Marshalla, Rod Jackson ${ }^{a}$ \\ ${ }^{a}$ Section of Epidemiology and Biostatistics, School of Population Health, Tamaki Campus, University of Auckland, Auckland, \\ New Zealand
}

Received 10th March 2010; revised 14th July 2010; accepted 18th September 2010; online 28th December 2010

\begin{abstract}
Aims: To examine the ecological association between population asthma symptom prevalence in six to seven year-old children and per capita sugar consumption seven years earlier (during the perinatal period).

Methods: The asthma data (from the International Study of Asthma and Allergies in Childhood [ISAAC] study) were collected between 1999 and 2004 from 53 countries, and per capita sugar consumption data (seven years before the asthma prevalence) were extracted from United Nations Food and Agriculture (UNFAO) food balance sheets. Linear regression and Spearman's rank coefficient were used to evaluate the relationship between exposure and disease outcome.

Results: Per capita sugar consumption varied more than six fold-between countries. A log-linear relationship was found between severe asthma symptoms (\%) and per capita added sugar consumption in kg/capita/year (exponentiated beta coefficient 1.020; $95 \% \mathrm{Cl} 1.005$ to 1.034; $P=0.012$ ). Spearman's rank correlation coefficient was $0.34(P=0.015)$, which indicates moderate correlation.

Conclusions: We have demonstrated an ecological association between sugar consumption during the perinatal period and subsequent risk of severe asthma symptoms in six and seven year-olds.

(C) 2011 Primary Care Respiratory Society UK. All rights reserved.

S Thornley et al. Prim Care Respir J 2011; 20(1): 75-78

doi:10.4104/pcrj.2010.00087
\end{abstract}

Keywords asthma, aetiology, ISAAC data, sugar consumption, observational study, nutrition

\section{Introduction}

The global prevalence of both obesity and asthma has increased substantially in the last forty years.' The co-occurrence of these two epidemics has led some investigators to propose that nutritional factors responsible for obesity may also cause asthma. ${ }^{2,3}$ The association between obesity and asthma at an individual level has been reported, and remains controversial. ${ }^{3}$ Links between obesity and asthma have been drawn in both children and adults and a number of hypotheses put forward to explain these links.

Recently, we proposed that increased refined carbohydrate intake may be responsible for increasing prevalence of obesity. ${ }^{4}$ Ecological analyses of country-level per capita food disappearance data on added sugar, from the routinely published United Nations Food and Agriculture (UNFAO) Food Balance Sheets, supports our hypothesis. ${ }^{5}$

The International Study of Asthma and Allergies in
Childhood (ISAAC) has generated individual level information on asthma symptoms and putative exposures from 463,801 children aged between 13 and 14 years and 257,800 children aged 6 and 7 in 58 countries. ${ }^{6}$ The authors reported considerable variation in the prevalence of asthma between countries (measured with a standardised definition), suggesting that potentially avoidable causes may be present.

The importance of exposures during the perinatal period has been highlighted for both obesity and asthma $a^{7.8}$ and several reports have shown that rapid growth after birth is associated with increased risk of asthma. ' We therefore linked UNFAO and ISAAC data from 53 countries to explore the possibility of an association between added sugar consumption and asthma symptom prevalence, linking country-level data on severe asthma symptoms in children aged 6-7 years with per capita added sugar consumption data in the same countries during the children's perinatal period, seven years earlier.

\footnotetext{
* Corresponding author: Dr Simon Thornley, Section of Epidemiology and Biostatistics, Level 4, School of Population Health,Tamaki Campus, The University of Auckland, Private Bag 92019, Auckland 1151, New Zealand. Tel: +6493737599 ext 81971 E-mail: s.thornley@auckland.ac.nz
} 


\section{Methods}

\section{Outcome data - asthma symptom prevalence}

The ISAAC study, Phase Three, generated asthma symptom prevalence information at country level, drawn from surveys of 6-7 and 13-14 year-old children, using a standardised questionnaire, from 58 countries. A number of indicators of asthma prevalence have been reported including current wheeze, parental- (6 to 7 year-olds) or self- (13 to 14 year-old) reported asthma, and symptoms of severe asthma. We used the 6-7 year-old age group with "symptoms of severe asthma" as our measure of asthma prevalence because we believed that this category would be most consistently reported between countries and may be less subject to measurement error than the other reported measures of "current wheeze" and the question "has your child ever had asthma?".

Symptoms of severe asthma included respondents with one or more of the following symptoms:

(1) $\geq 4$ attacks of wheeze in the last year, or,

(2) $\geq$ one or more nights per week sleep disturbance from wheeze in the last year, or,

(3) wheeze affecting speech in the last year.

Published prevalence estimates for these countries were derived from surveys carried out between 1999 and $2004 .^{6}$ Although we have reported country estimates, these surveys were taken from specific localities within countries - and in some cases, several regional study results have been pooled to provide a total country estimate.

\section{Exposure data - added sugar consumption}

We used the United Nations Food and Agricultural Organisation (UNFAO) food balance sheets ${ }^{9}$ to estimate per capita consumption of a variety of simple carbohydrates. We used the UNFAO category "sugar and sweeteners" which includes a variety of monosaccharides and disaccharides which mainly consist of sucrose (from sugar beet or cane), glucose, honey or high fructose corn syrup ${ }^{5}$ referred to here as "added sugar". Such foods are high in fructose concentration, either free or as part of a disaccharide (i.e. sucrose). This nutrient exposure information was extracted from the period when the mothers of the children surveyed for asthma symptoms were most likely to be pregnant (seven years before the asthma survey was undertaken). Per capita added sugar consumption is calculated by taking the difference of quantity of the sum of added sugar imported and produced from total added sugar exported or used for industry. The quantity that remains has therefore 'disappeared' and is assumed to have been consumed. Food balance sheet data, therefore, does not account for food either not consumed or wasted.

\section{Data analysis}

We linked the exposure and outcome data using linear regression and Spearman's rank correlation coefficient. Asthma symptom prevalence was log transformed to stabilise its variance. The R-project was used for all analyses. ${ }^{10}$

\section{Results}

We found estimates of severe asthma symptom prevalence for 58 countries from ISAAC publications, but five of these countries did not appear in UNFAO food balance records, leaving 53 countries with paired data for analysis. Symptom prevalence ranged from $1.1 \%$ in Indonesia to $20.3 \%$ in Costa Rica. Sample sizes of surveys ranged from 1,181 (Bulgaria - one survey) to 46,761 (India - pooled estimate). Severe asthma symptom prevalence was generally higher in English speaking and Latin

Figure 1. Severe asthma symptom prevalence in 6 and 7 year-old children plotted against average per capita added sugar consumption, 7 years

prior, by country. Solid line shows log-linear regression line with dashed lines illustrating $95 \%$ confidence intervals.

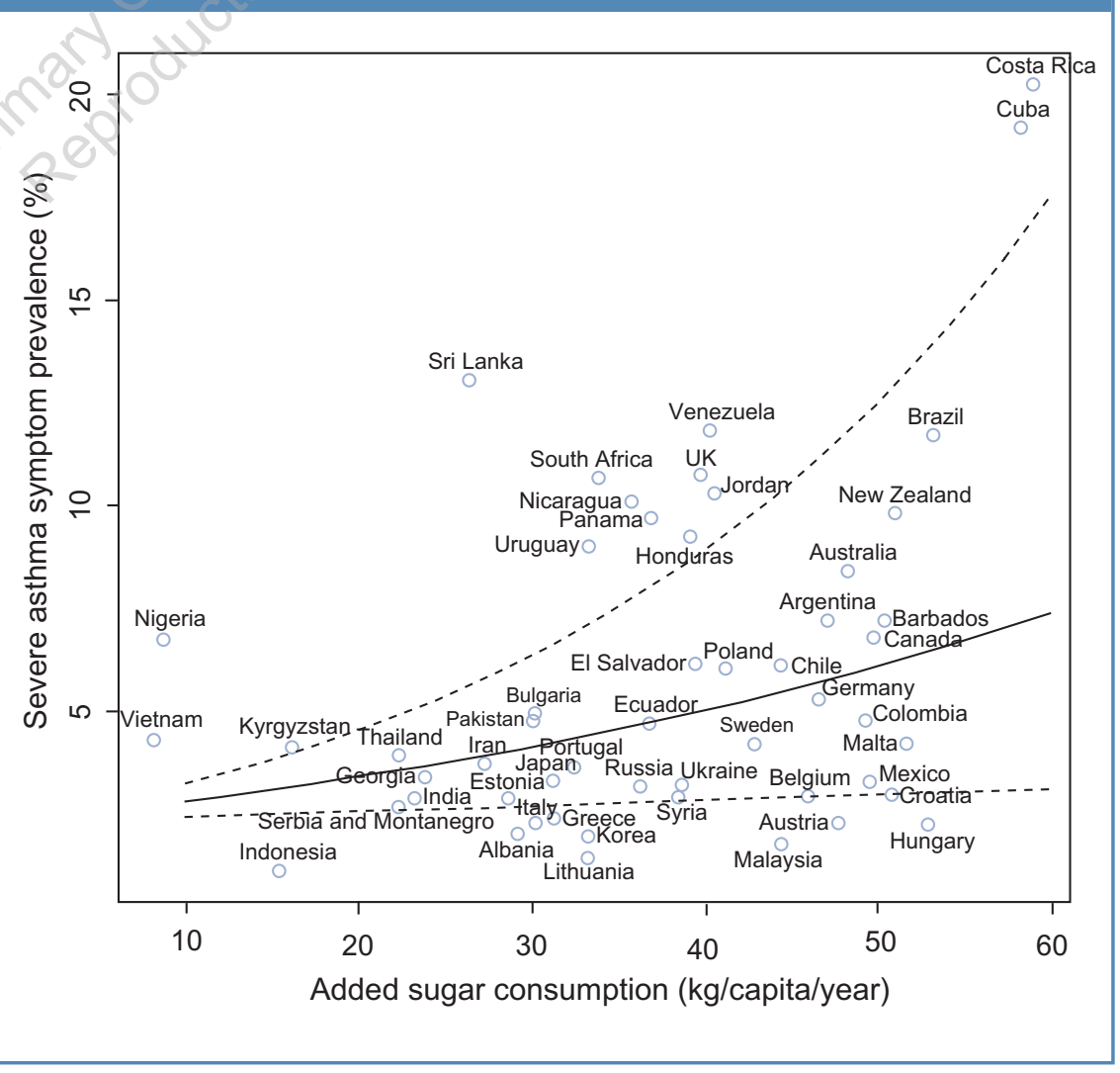


American countries, with lower estimates in Asia, Eastern European and Mediterranean countries. Per capita added sugar consumption recorded seven years before the asthma surveys varied more than six-fold, with lowest average consumption in Vietnam (8.15 kg/capita/year in 1994) and highest in Costa Rica (58.95 kg/capita/year in 1995) and Cuba (58.23 kg/capita/year in 1995), two sugar exporting nations. The bivariate distribution of the data is displayed in Figure 1. A log-linear relationship was found between severe asthma symptoms (\%) and per capita sugar consumption in $\mathrm{kg} / \mathrm{capita} /$ year (exponentiated beta coefficient 1.020; $95 \% \mathrm{Cl} 1.005$ to 1.034; $\mathrm{P}=0.012$ [estimate and $95 \% \mathrm{Cl}$ displayed in Figure 1]). The model indicates that centres with a further $35 \mathrm{~kg} / \mathrm{capita} / \mathrm{year}$ greater added sugar disappearance have double the symptom prevalence compared to a country with lesser consumption. Although most countries occupy the area within the bounds of uncertainty of the regression estimate, some outliers exist such as Nigeria and Sri Lanka, with unexpectedly high prevalence given their average sugar consumption. Conversely, Hungary, Austria and Malaysia have low rates of asthma at relatively high levels of sugar consumption. Plots of residual versus predicted values indicated acceptable model fit. When data from two high outliers (Costa Rica and (uba) were excluded, the magnitude of the exponentiated beta coefficient reduced to $1.013(95 \% \mathrm{Cl} 0.998$ to $1.028 ; \mathrm{P}=0.104)$.

Our regression analysis implies directionality, so that average between-country asthma symptom prevalence is estimated from observed values of added sugar consumption, which reflects our a priori belief that increased sugar consumption influences asthma symptom prevalence. Spearman's rho rank correlation coefficient, in contrast, is an unconditional measure of association, estimated at $0.34(P=0.015)$ - which indicates moderate correlation between the two variables.

\section{Discussion}

Per capita added sugar consumption (an indirect indicator of perinatal sugar exposure) estimated from FAO food balance sheets, positively correlated with between-country level estimates of severe asthma symptom prevalence in 6 and 7 year-olds, measured seven years later. Of note, the two countries with the highest recorded prevalence of severe asthma symptoms also had the highest average rate of sugar consumption. This association explains the pattern of high rates of childhood asthma in English speaking and Latin American countries. Also, the countries with the top five highest prevalence of severe asthma in this age group (Costa Rica, Cuba, Sri Lanka, Venzuela and Brazil) all have local sugar manufacturing industries.

Our analysis, like all ecological studies, is limited by the use of aggregate data. These types of studies are prone to measurement error and confounding, but nevertheless have previously identified many causal associations. We have linked an estimate of total per capita added sugar disappearance at a country level to an estimate of asthma symptom prevalence from either one or an aggregation of regional population samples for a defined age group in these countries. We do not know if the relative or total consumption of added sugar in pregnant women is likely to be similar to the population average. The accuracy of food disappearance data to measure actual food intake is also unknown. Previous studies have focused on the use of this data to estimate average and relative fat intakes." Another limitation of our analysis was the exclusion of the United States (USA), which did not report asthma symptom prevalence in this phase of the ISAAC study. If included, the USA had the highest per capita sugar consumption of any country in our cohort, with 66.4 $\mathrm{kg} /$ capita/year consumed in 1994. In earlier phases of the ISAAC study, the USA prevalence of asthma in 13 to 14 year old children was about $20 \%$ or 8 th out of 56 surveyed countries, immediately below Brazil. ${ }^{12}$ If the USA had a similar between-country rank for 6 to 7 year-old severe asthma symptoms, the inclusion of this data point would further strengthen the relationship we found between other countries.

A strength of this study lies in the use of the ISAAC study information, which has provided the most comprehensive, standardised, between-country attempt to estimate global asthma prevalence in children to date. Also, the ubiquitous nature of sweeteners in modern diets suggests that our study is less likely to suffer from confounding related directly or indirectly to food choices. Our sensitivity analysis, which excluded two outliers, showed that a positive relationship was still observed between the variables, although the strength of the association was diminished.

Despite multiple possible sources of error, we have demonstrated a correlation between these two variables, which is unlikely to be due to chance alone. These findings were not part of a 'data trawling' exercise but represent the first joint analyses of these datasets by the first author (ST), who is developing a research program on the relationship between refined carbohydrates and disease. Also, epidemiological evidence of rising sugar consumption, ${ }^{5}$ the rising prevalence of obesity, ${ }^{1}$ and the reported association between asthma and obesity ${ }^{2}$ at an individual level make a plausible argument to link these disorders.

Although these trends coincide, we can only speculate on a biological mechanism that may link sugar consumption and childhood asthma. ${ }^{5}$ The generally accepted co-occurrence of obesity and asthma is still poorly understood at a physiological level. Adipose tissue secretes hormones such as leptin, which induces satiety in the hypothalamus but also modulates the function of T-helper immune cells. ${ }^{13}$ Other disorders that are linked with the immunocompromised state - such as a range of 


\section{Summary \\ This study shows a moderate association between per capita sugar consumption during the perinatal period and subsequent prevalence of severe childhood asthma symptoms. This association explains the pattern of high rates of childhood asthma in English speaking and Latin American countries. Cohort studies that collect detailed nutrient information, may further investigate the strength of this association. If sugar consumption is causally associated with the onset of childhood asthma, restricting intake of this substance is likely to improve symptoms and reduce frequency of exacerbations in people with established disease.}

cancers - are also linked to obesity. ${ }^{14}$ However, the mechanism by which obesity and asthma are linked is yet to be determined. ${ }^{13,15}$

To our knowledge, this is the only study so far to link sugar or fructose with asthma symptom prevalence. Although previous studies have attempted to uncover a dietary link between childhood asthma and dietary factors, most studies have focused on the role of different sub-types of fats, vitamins or trace elements, ${ }^{13}$ none of which show persuasive evidence of an association, let alone causation. Other studies have implicated the importance of perinatal exposures; again; however, no consistent evidence for a causative mechanism has been forthcoming. Our analyses were based on an a priori hypothesis, but we did check the association between asthma symptom prevalence and sugar consumption measured over the same period. A lesser association was observed (exponentiated beta coefficient 1.015; $95 \% \mathrm{Cl} 1.029$ to 1.001; $\mathrm{P}=0.038$; Spearman's correlation coefficient 0.24 ). This finding strengthens the argument that a latency period (birth to 6-7 years-old chosen in this study) is necessary for sugar consumption to influence asthma prevalence.

If our observed association is causal, and sugar consumption plays a role in the aetiology of asthma, we also expect that limiting sugar consumption in people with established asthma may result in clinical benefit. To our knowledge, no randomised controlled trials have been conducted to test such a hypothesis. One Singaporean observational study of mothers of children with asthma showed that five of the most commonly reported food items thought to exacerbate the condition had high levels of added sugar, and the second most commonly reported food was "fizzy drinks". ${ }^{16}$ Given that sugar (sucrose) has little nutritional value, few adverse effects are likely to result from dietary advice to restrict sugar consumption in people with established asthma.
Although by no means confirmatory, this study raises the possibility of a link between increasing global sugar consumption and asthma prevalence. Longitudinal studies both observational and experimental - using individual level information are needed to investigate further the strength of this association.

\section{Conflict of interest declarations}

None.

\section{Funding}

This work was funded by a salaried position at the University of Auckland

\section{References}

1. OECD, OECD Health Data 2009: Statistics and Indicators for 30 Countries. 2009, OECD publishing: Paris.

2. Deane S, Thomson A. Obesity and the pulmonologist. Arch Dis Child 2006 91(2):188-91. http://dx.doi.org/10.1136/adc.2005.072223

3. Ford ES. The epidemiology of obesity and asthma. Journal of Allergy and Clinical Immunology 2005;115(5):897-909. http://dx.doi.org/10.1016/ j.jaci.2004.11.050

4. Thornley S, McRobbie H, Eyles H, Walker N, Simmons $G$. The obesity epidemic: is glycemic index the key to unlocking a hidden addiction? Medical Hypotheses 2008;71(5):709-14. http://dx.doi.org/10.1016/j.mehy.2008.07.006

5. Popkin BM, SJ Nielsen. The sweetening of the world's diet. Obesity Research 2003;11(11):1325-32. http://dx.doi.org/10.1038/oby.2003.179

6. Lai CK, Beasley R, Crane J, Foliaki S, Shah J, Weiland S. Global variation in the prevalence and severity of asthma symptoms: phase three of the International Study of Asthma and Allergies in Childhood (ISAAC). Thorax 2009;64(6):47683. http://dx.doi.org/10.1136/thx.2008.106609

7. Litonjua AA, Gold DR. Asthma and obesity: common early-life influences in the inception of disease. J Allergy Clin Immunol 2008;121(5):1075-84; quiz 10856. http://dx.doi.org/10.1016/j.jaci.2008.03.005

8. Gilliland FD, Berhane K, Islam T, et al. Obesity and the Risk of Newly Diagnosed Asthma in School-age Children. Am J Epidemiol 2003;158(5):406-15. doi:10.1093/aje/kwg175

9. United Nations Food and Agriculture Organisation. FAOSTAT. 2009 [cited 2009 4 November]; Available from: http://faostat.fao.org/site/291/default.aspx.

10. R Development Core Team, R: A language and environment for statistical computing. 2007, R Foundation for Statistical Computing: Vienna.

11. Laugesen M, Swinburn B. The New Zealand food supply and diet--trends $1961-$ 95 and comparison with other OECD countries. Organisation for Economic Cooperation and Development. N Z Med J 2000;113(1114):311-15.

12. Beasley R. The burden of asthma with specific reference to the United States. J Allergy Clin Immunol 2002;109(5):S482-S489. http://dx.doi.org/10.1067/ mai.2002.122716

13. Tantisira KG, Weiss ST. Complex interactions in complex traits: obesity and asthma. Thorax 2001; 56(90002):ii64-74.

14. Bianchini F, Kaaks R, Vainio H. Overweight, obesity, and cancer risk. The Lancet Oncology 2002;3(9):565-74. http://dx.doi.org/10.1016/S1470-2045(02)00849-5

15. Weiss ST, Shore S. Obesity and Asthma: Directions for Research. Am J Respir Crit Care Med 2004;169(8):963-8. http://dx.doi.org/10.1164/rccm.200303403WS

16. Ang K, Foo S. Childhood asthma and dietary management among Singapore parents. Nutrition \& Food Science 2001;31(6):310-35.

\section{Available online at http://www.thepcrj.org}

